\section{DETECTION OF BILIARY FUNCTIONAL PROBLEMS USING MICROWAVES}

\author{
Anil Lonappan, ${ }^{1}$ Benzy Paul, ${ }^{2}$ V. O. Thimothy, ${ }^{3}$ \\ C. Rajasekaran, ${ }^{4}$ Vinu Thomas, ${ }^{1}$ Joe Jacob, ${ }^{1}$ and \\ K. T. Mathew ${ }^{1}$ \\ ${ }^{1}$ Department of Electronics, Microwave Tomography and Materials \\ Research Laboratory, Cochin University of Science and Technology, \\ Kochi 682 022, India; Corresponding author: anil@cusat.ac.in \\ 2 Department of Pathology, Mahatma Gandhi Mission's Medical \\ College, Kamothe, Navi Mumbai 410 209, India \\ ${ }^{3}$ Department of Surgery, Amala Institute of Medical Sciences and \\ Research Centre, Amala Nagar, Thrissur 680 555, India \\ ${ }^{4}$ Department of Medicine, Medical College, Trivandrum 695011 , \\ India
}

Received 25 May 2008

\begin{abstract}
This article reports a new in vitro bile analysis based on the measurement of the dielectric properties at microwave frequencies. The measurements were made using rectangular cavity perturbation technique at the S-band of microwave frequency with the different samples of bile obtained from healthy persons as well as from patients. It is observed that an appreciable change in the dielectric properties of patient's samples with the normal healthy samples and these measurements were in good agreement with clinical analysis. These results prove an alternative in-vitro method of detecting bile abnormalities based on the measurement of the dielectric properties of bile samples using microwaves without surgical procedure. () 2008 Wiley Periodicals, Inc. Microwave Opt Technol Lett 51: 101-103, 2009; Published online in Wiley InterScience (www.interscience.wiley.com). DOI 10.1002/mop. 24021
\end{abstract}

Key words: bile; liver; gall bladder; cavity perturbation; clinical analysis

\section{INTRODUCTION}

Bile is a bitter, yellow or green alkaline fluid secreted by hepatocytes and its constituents are water, cholesterol, bile pigments, bicarbonate ions, and bile salts. It is stored in the gallbladder between meals and while eating is discharged into the duodenum. The gall bladder absorbs the water and electrolytes concentrating it between the meals and when the chyme enters the small intestine, it excretes waste and aids the process of digestion of lipids. Cholesterol is also released with the bile. The human liver can produce close to 11 of bile per day depending on the body size. Cholelithiasis is known as gallstones which are formed in the gallbladder due to variation in concentration of bile constituents. Choledocholithiasis is the presence of gallstones in the common bile duct [1]. Cholestatic jaundice is caused by thickened bile or bile plugs in the small biliary passages of the liver. These situations can lead to the gall bladder rupture or form an abscess which leads to a life-threatening infection of the liver requires medical emergency, the endoscopic retrograde cholangiopancreatography (ERCP) procedure, or surgical treatment. This article reports a comprehensive study of the dielectric properties of human bile at microwave frequencies. The samples were collected from healthy persons and people ailing from cholelithiasis, choledocholithiasis, and cholestatic jaundice. The measurement of the dielectric properties of bile helps to identify the healthy condition as well as diseased condition as there is significant variation in the dielectric properties.

Biological effects of microwaves and the application of microwaves in medicine are developing areas of research [2]. The nonionizing microwave radiation interacts with tissues and obtains a large dielectric contrast according to their water content. Thus, there is a need to study the interaction of microwave with tissues especially its effect on biological materials. The key element in the microwave study is the determination of the absorbed energy. The amount of energy absorbed is a function of the complex permittivity of a material [3]. Hence, it is crucial to know the dielectric properties of biological materials and the various constituents thereof. Exhaustive studies of dielectric parameters of various human tissues and body fluids at different RF frequencies have been reported [4-6]. Different measurement techniques can be adopted to measure the complex permittivity of a material and the chosen technique depends on various factors such as the nature of the sample and the frequency range used [7-10]. When only very small volumes of the sample are available, the cavity perturbation technique is an attractive option as it requires only minute volumes for the measurement [11]. This makes it suitable for the dielectric study of bile as only very small volumes are needed to be extracted by the procedure. However, no data are available for the complex permittivity of bile in the literature. The rectangular cavity perturbation technique has been employed for the measurement of the dielectric parameters of bile obtained from healthy persons as well as from patients in this work, in the frequency range $2-3 \mathrm{GHz}$. It is noticed that a remarkable change in the dielectric properties of patient samples with the normal healthy samples and these measurements were in good agreement with clinical analysis. This microwave measurement procedure is simple and extraction of bile from persons is least painful and nonsurgical in nature. These results prove an alternative in-vitro method of detecting bile abnormalities based on the measurement of the dielectric properties of bile samples using microwaves without surgical procedure.

\section{SAMPLE PREPARATION}

Bile is collected by means of upper gastrointestinal endoscope by placing it into the descending duodenum called the papilla of vater where bile duct and pancreatic duct opens into gastrointestinal tract. The bile samples collected were then filled in the sample holder and kept at $1^{\circ} \mathrm{C}$. Measurements were carried out on samples which were less than 1 day old.

\section{PROCEDURE}

The experimental set-up consists of a transmission type S-band rectangular cavity resonator, HP 8714 ET network analyzer. The cavity resonator is a transmission line with one or both ends closed. The numbers of resonant frequencies are determined by the length of the resonator. The resonator in this set-up is excited in the $\mathrm{TE}_{10 \rho}$ mode. The sample holder which is made of glass in the form of a capillary tube flared to a disk shaped bulb at the bottom is placed into the cavity through the nonradiating cavity slot, at broader side of the cavity which can facilitate the easy movement of the holder. The resonant frequency $f_{\mathrm{o}}$ and the corresponding quality factor $Q_{\mathrm{o}}$ of the cavity at each resonant peak with the empty sample holder placed at the maximum electric field are noted. The same holder filled with known amount of sample under study is again introduced into the cavity resonator through the nonradiating slot. The resonant frequencies of the sample loaded cavity are selected and the position of the sample is adjusted for maximum perturbation (i.e., maximum shift of resonant frequency with minimum amplitude for the peak). The new resonant frequency $f_{\mathrm{s}}$ and the quality factor $Q_{\mathrm{s}}$ are noted. The same procedure is repeated for other resonant frequencies.

\section{THEORY}

When a material is introduced into a resonant cavity, the cavity field distribution and resonant frequency are changed which de- 
pend on shape, electromagnetic properties, and its position in the fields of the cavity. Dielectric material interacts only with electric field in the cavity.

According to the theory of cavity perturbation, the complex frequency shift is related as [11]

$$
\begin{array}{r}
-\frac{d \Omega}{\Omega} \approx \frac{\left(\bar{\varepsilon}_{\mathrm{r}}-1\right) \int_{V_{\mathrm{s}}} E \cdot E_{0}^{*} d V}{2 \int\left|E_{0}\right|^{2} d \mathrm{~V}}, \\
\text { but } \frac{d \Omega}{\Omega} \approx \frac{d \omega}{\omega}+\frac{i}{2}\left[\frac{1}{Q_{s}}-\frac{1}{Q_{0}}\right]
\end{array}
$$

Equating (1) and (2) and separating real and imaginary parts results

$$
\begin{aligned}
& \varepsilon_{\mathrm{r}}^{\prime}-1=\frac{f_{\mathrm{o}}-f_{\mathrm{s}}}{2 f_{\mathrm{s}}}\left(\frac{V_{\mathrm{c}}}{V_{\mathrm{s}}}\right), \\
& \varepsilon_{\mathrm{r}}^{\prime \prime}=\frac{V_{\mathrm{c}}}{4 V_{\mathrm{s}}}\left(\frac{Q_{\mathrm{o}}-Q_{\mathrm{s}}}{Q_{\mathrm{o}} Q_{\mathrm{s}}}\right) .
\end{aligned}
$$

Here, $\varepsilon_{\mathrm{r}}=\varepsilon_{\mathrm{r}}^{\prime}-j \varepsilon_{\mathrm{r}}^{\prime \prime}, \varepsilon_{\mathrm{r}}$ is the relative complex permittivity of the sample, $\varepsilon_{\mathrm{r}}^{\prime}$ is the real part of the relative complex permittivity, which is known as dielectric constant. $\varepsilon_{\mathrm{r}}^{\prime \prime}$ is the imaginary part of the relative complex permittivity associated with the dielectric loss of the material. $V_{\mathrm{s}}$ and $V_{\mathrm{c}}$ are corresponding volumes of the sample and the cavity resonator. The conductivity can be related to the imaginary part of the complex dielectric constant as

$$
\sigma_{\mathrm{e}}=\omega \varepsilon^{\prime \prime}=2 \pi f \varepsilon_{0} \varepsilon_{\mathrm{r}}^{\prime \prime}
$$

\section{RESULTS AND DISCUSSION}

Bile samples are collected from healthy donors as well as from the patients. Clinical procedures are performed in the medical laboratories to confirm the disease of a patient and report containing the clinical parameters is given which depend on the type of disease. The clinical laboratory parameter and the dielectric constant of bile are compared to obtain a relation.

Cholelithiasis is known as gallstones which are formed in the gallbladder due to variation in concentration of bile constituents. Gallstones can occur anywhere within the biliary tree, including the gallbladder and the common bile duct. Table 1 shows the variation of normal healthy bile samples with that cholelithiasis

TABLE 1 Variation of Constituents in Normal Healthy Bile Samples and Cholelithiasis Samples

\begin{tabular}{lcccc}
\hline Sample & $\begin{array}{c}\text { Cholesterol } \\
(\mathrm{mg} / \mathrm{dl})\end{array}$ & $\begin{array}{c}\text { Bile Pigments } \\
(\mathrm{mg} / \mathrm{dl})\end{array}$ & $\begin{array}{c}\text { Bicarbonate Ions } \\
(\mathrm{mg} / \mathrm{dl})\end{array}$ & $\begin{array}{c}\text { Bile Salts } \\
(\mathrm{mg} / \mathrm{dl})\end{array}$ \\
\hline $\mathrm{bN}-1$ & $170-195$ & $50-95$ & $28-40$ & $32-66$ \\
$\mathrm{bN}-2$ & $168-205$ & $45-88$ & $32-45$ & $40-60$ \\
$\mathrm{bN}-3$ & $160-185$ & $69-78$ & $40-56$ & $25-45$ \\
$\mathrm{bN}-4$ & $155-180$ & $65-85$ & $38-54$ & $30-58$ \\
$\mathrm{bCHO}-1$ & $720-922$ & $220-240$ & $250-310$ & $150-190$ \\
$\mathrm{bCHO}-2$ & $868-1005$ & $318-342$ & $240-305$ & $164-204$ \\
$\mathrm{bCHO}-3$ & $760-845$ & $232-240$ & $262-290$ & $160-198$ \\
$\mathrm{bCHO}-4$ & $855-910$ & $275-330$ & $220-270$ & $150-210$ \\
\hline
\end{tabular}

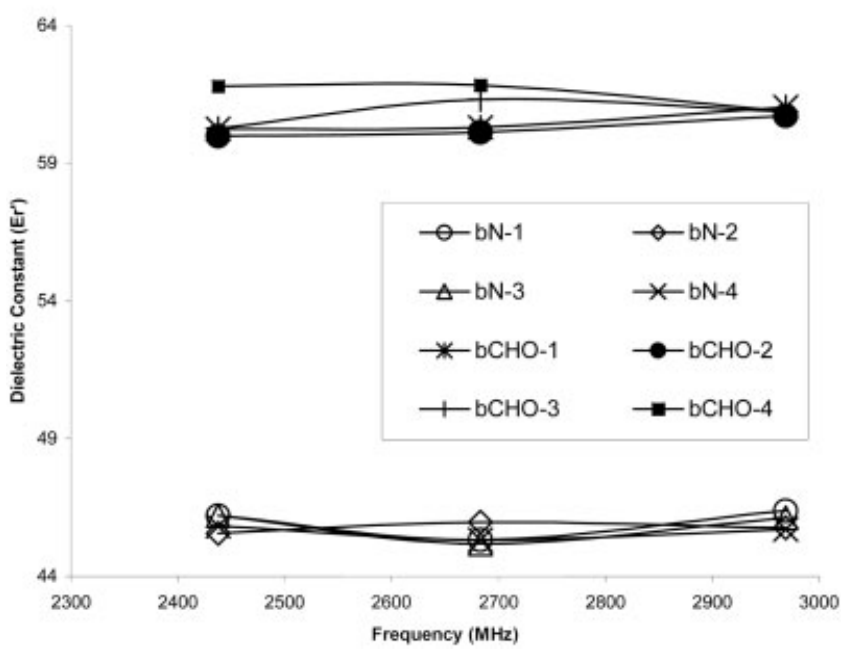

Figure 1 Variation of dielectric constant in normal healthy bile samples and cholelithiasis samples

samples and is observed that there is a significant variation in the constituents of normal bile samples and cholelithiasis bile samples. The variation of the dielectric constants of normal bile samples and cholelithiasis bile samples is shown in Figure 1. This shows that dielectric constant of cholelithiasis bile samples is more than that of normal bile samples. Figure 2 shows that conductivity of cholelithiasis bile samples is more than that of normal bile samples and the increased level of bicarbonate ions and bile salts.

Cholestatic jaundice is caused by thickened bile or bile plugs in the small biliary passages of the liver. Table 2 shows the variation of normal healthy bile samples with that cholestatic jaundice samples and is observed that there is a significant variation in the constituents of normal bile samples and cholestatic jaundice bile samples. The variation of the dielectric constants of normal bile samples and cholestatic jaundice bile samples is shown in Figure 3. This shows that dielectric constant of cholestatic jaundice bile samples is more than that of normal bile samples. Figure 4 shows that conductivity of cholestatic jaundice bile samples is more than

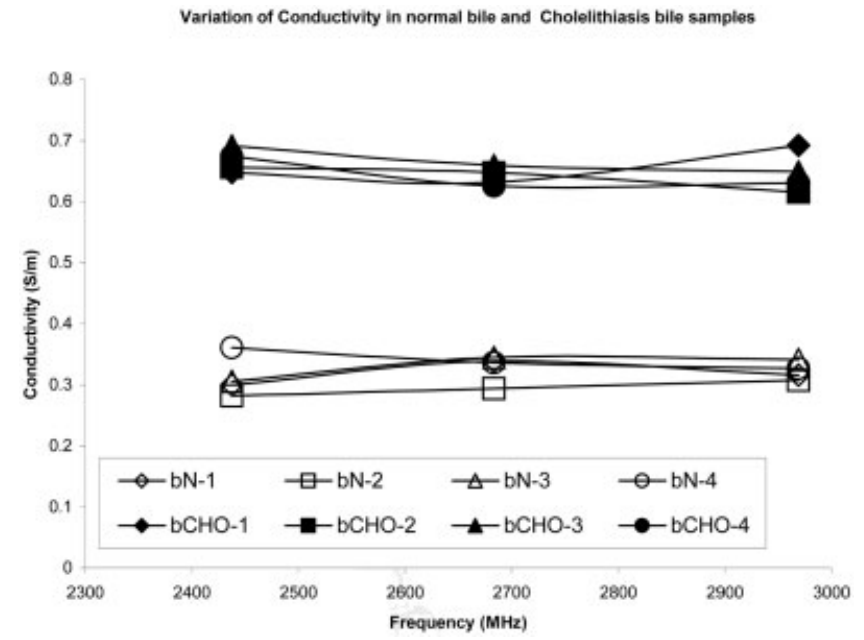

Figure 2 Variation of conductivities in normal bile and cholelithiasis bile samples 
TABLE 2 Variation of Constituents in Normal Healthy Bile Samples and Cholestatic Jaundice Samples

\begin{tabular}{lcccc}
\hline Sample & $\begin{array}{c}\text { Cholesterol } \\
(\mathrm{mg} / \mathrm{dl})\end{array}$ & $\begin{array}{c}\text { Bile Pigments } \\
(\mathrm{mg} / \mathrm{dl})\end{array}$ & $\begin{array}{c}\text { Bicarbonate Ions } \\
(\mathrm{mg} / \mathrm{dl})\end{array}$ & $\begin{array}{c}\text { Bile Salts } \\
(\mathrm{mg} / \mathrm{dL})\end{array}$ \\
\hline $\mathrm{bN}-1$ & $170-195$ & $50-95$ & $28-40$ & $32-66$ \\
$\mathrm{bN}-2$ & $168-205$ & $45-88$ & $32-45$ & $40-60$ \\
$\mathrm{bN}-3$ & $160-185$ & $69-78$ & $40-56$ & $25-45$ \\
bN-4 & $155-180$ & $65-85$ & $38-54$ & $30-58$ \\
bCJ-1 & $412-440$ & $242-322$ & $322-373$ & $129-135$ \\
bCJ-2 & $423-461$ & $205-292$ & $307-327$ & $114-127$ \\
bCJ-3 & $440-476$ & $244-273$ & $346-360$ & $122-140$ \\
bCJ-4 & $413-455$ & $269-292$ & $356-377$ & $130-138$ \\
\hline
\end{tabular}

that of normal bile samples and the increased level of bicarbonate ions and bile salts.

The results of the case study have shown a great correlation between the laboratory results and bile diagnosis using microwaves.

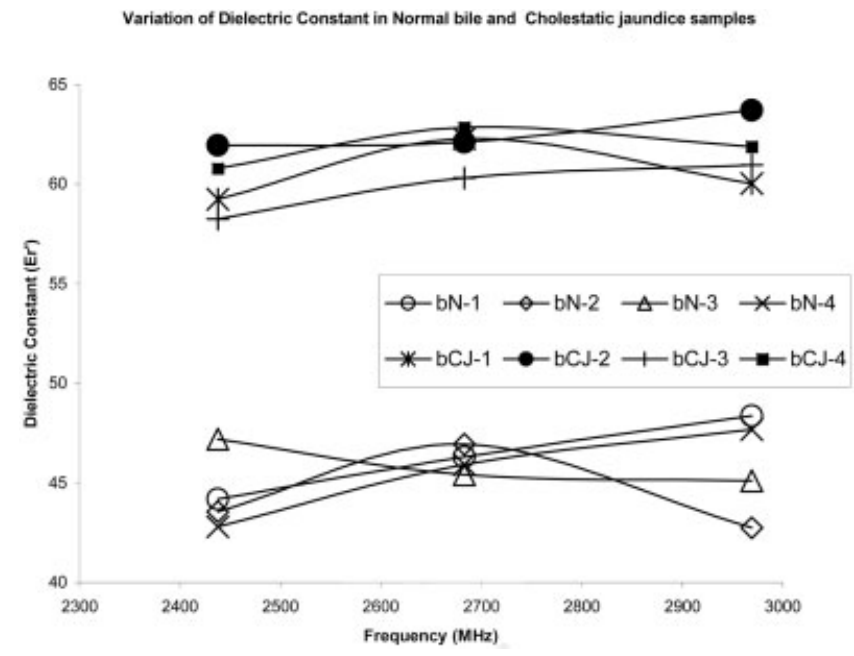

Figure 3 Variation of dielectric constant in normal healthy bile samples and cholestatic jaundice samples

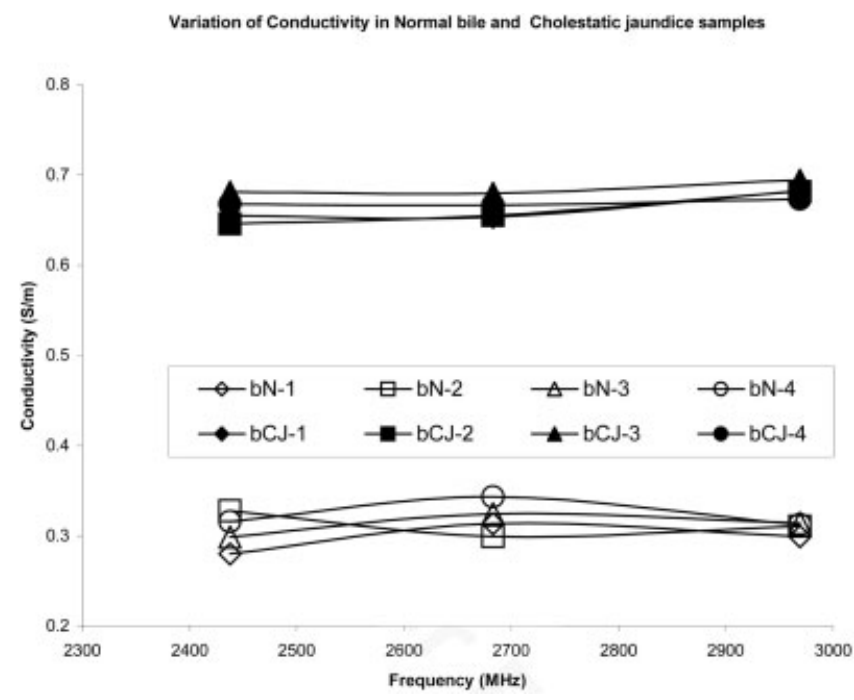

Figure 4 Variation of conductivities in normal bile and cholestatic jaundice samples

\section{CONCLUSION}

The microwave characterization of the bile samples is done using cavity perturbation technique. The cavity perturbation technique is quick, simple, and accurate and it requires very low volume of sample for measuring the dielectric properties of tissue samples and biological fluids. It is observed that in the specified band of frequencies, there is an appreciable change in the dielectric properties of patient samples with the normal healthy samples. These results prove an alternative in-vitro method of detecting bile abnormalities based on the measurement of the dielectric properties of bile samples using microwaves without surgical procedure.

\section{REFERENCES}

1. A. Lauri, R.C. Horton, B.R. Davidson, A.K. Burroughs, and J.S. Dooley, Endoscopic extraction of bile duct stones: management related to stone size, Gut 34 (1993), 1718-1721.

2. "Special Issues," IEEE Trans Microwave Theory Tech 50 (2002).

3. A. Von Hippel, Dielectric and waves, Artech House, Norwood, MA, 1995.

4. S. Gabriel, R.W. Lau, and C. Gabriel, The dielectric properties of biological tissues. II. Measurements on the frequency range $10 \mathrm{~Hz}$ to $20 \mathrm{GHz}$ Literature survey, Phys Med Biol 41 (1996), 2251-2269.

5. H.F. Cook, Dielectric behavior of human blood at microwave frequencies, Nature 168 (1951), 247-248.

6. H.F. Cook, The dielectric behavior of some types of human tissues at microwave frequencies, Br J Appl Phys 2 (1951), 295-300.

7. D.K. Ghodgaonkar, V.V. Varadan, and V.K. Varadan, Free space measurement of complex permittivity and complex permeability of magnetic materials at microwave frequencies, IEEE Trans Instrument Measurement 19 (1990), 387-394.

8. D.K. Ghodgaonkar, V.V. Varadan, and V.K. Varadan, A free space method for measurement of dielectric constant and loss tangents at microwave frequencies, IEEE Trans Instrument Measurement 38 (1989), 789-793.

9. W. Barry, A broadband, automated, stripline technique for the simultaneous measurement of complex permittivity and complex permeability, IEEE Trans Microwave Theory Tech 34 (1986), 80-84.

10. Z. Abbas, R.D. Pollard, and R.W. Kelsall, A rectangular dielectric waveguide technique for determination of permittivity of materials at W-band, IEEE Trans Microwave Theory Tech 46 (1998), 2011-2015.

11. K.T. Mathew, Perturbation theory, Encyclopedia of RF and microwave engineering, Vol. 4, Wiley-Interscience, USA, 2005, pp 3725-3735.

(c) 2008 Wiley Periodicals, Inc.

\section{WWAN CERAMIC CHIP ANTENNA FOR MOBILE PHONE APPLICATION}

\section{Ming-Ren Hsu and Kin-Lu Wong}

Department of Electrical Engineering National Sun Yat-Sen University, Kaohsiung 804, Taiwan; Corresponding author:

hsumr@ema.ee.nsysu.edu.tw

\section{Received 26 May 2008}

ABSTRACT: A promising compact ceramic chip antenna capable of generating two wide operating bands at about 900 and $2000 \mathrm{MHz}$ for covering GSM850/900/1800/1900/UMTS WWAN (wireless wide area network) operation is presented. The antenna comprises a ceramic chip base of high relative permittivity 40 and small volume $2.5 \times 5 \times 40$ $\mathrm{mm}^{3}\left(0.5 \mathrm{~cm}^{3}\right)$ and a simple metal pattern embedded therein. The metal pattern is of an asymmetric T-shape with two different simple radiating arms; no meandering in the metal pattern is used, which is different from the meandered-type metal pattern used in conventional chip antennas. Without meandering in the metal pattern, the possible large coupling between the adjacent portions in the metal pattern can be avoided. 